\title{
SPACE DISTRIBUTION OF X-RAY CLUSTERS OF GALAXIES
}

\author{
A. Cavaliere, II Università di Roma \\ S. Colafrancesco, Universitd di Padova
}

The Intra-Cluster Plasma constitutes an archive of the past history of all clusters and of many groups of galaxies: statistical observations of their X-ray emission will set significant constraints on the dynamical, thermal and chemical events in these cosmic structures. Data on the local X-ray luminosity function $N(L, z \simeq 0)$ were provided by the 1st generation X-ray surveys. HEAO II provided an integral of $N(L, z)$ out to $z \simeq 0.4$, the counts from a subsample of the MSS (Gioia et al. 1984, Ap.J. 283, 495): these counts result very flat, cf. Fig. 2.

We discuss these observations in the framework of the hierarchical clustering scenario for the dynamical structures, coupled with a two-components ICP: the average objects luminosity $L \propto$ $M \rho T^{1 / 2} *\left[f_{p}+\left(1-f_{p}\right) g(t)\right]^{2}$ depends also on the primordial ICP mass fraction $f_{p}$ and on the accumulated fraction $g(t)$. In the extreme case $f_{p} \rightarrow 1$, the ICP is dominated by "primordial" gas (i.e. preceding the group era). At the other extreme $f_{p} \rightarrow 0$, dominant is the mass produced by stars and lost by galaxies following an initial burst of star formation, $g(t) \propto t^{\xi}(\xi \lesssim 2 / 3)$, or from a more even SFR, $g(t) \propto t$.

Figs. 1, 2 highlight some of our results, computed from $\partial N / \partial t+\partial(\dot{L} N) / \partial L=S(L, t) ; S$ is the t-derivative of a Press \& Schechter-like multiplicity function, and $\dot{L}$ derives from $L$ above.

These results are understood on taking over from the simple scaling laws used by White (1982, in Morph. \& Dyn. of Galaxies) and by Kaiser (1986, M.N.R.A.S. 222, 323). The l.f. scales as $N(L, z) \sim N_{0}\left[L / L_{c}(z)\right] / M_{c} L_{c}$, comprising a density evolution and a luminosity anti-evolution. The counts tend to be flattened at high fluxes by the current formation of rich clusters (cfr. Cavaliere et al. 1986, Ap.J. 305, 651). At lower fluxes the d. evolution of poor clusters and groups [number $\left.\propto 1 / M_{c} \propto(1+z)^{6 /(n+3)}\right]$ is balanced by the 1 . effect $L_{c} \propto(1+z)^{(5+7 n) / 2(n+3)} *(1+z)^{-3 \xi}$. The counts tend to nearly Euclidean slope when $f_{p} \rightarrow 1$. But when $f_{p} \rightarrow 0, L_{c}$ decreased sharply into the past: the lower ICP mass in older groups did offset any hierarchical trend to higher total densities. The net balance tilts toward flat counts for any $n \gtrsim-2$, if $\xi \gtrsim 1 / 2$.

If the counts will remain so flat with statistics increasing, their slope will require $f_{p} \lesssim 0.2$ (see Fig. 2); $f_{p}>0.1$ is required by dissipative collapse of galaxies: the slope of the counts will constrain the efficiency of star formation (high) and the rate of galaxy evolution (continuous). Once $f_{p}$ is assessed, finer information can be derived concerning not only the ICP age, but also cosmogony: e.g., flatter initial perturbation spectra and/or a larger variance in their probability distribution flatten the luminosity functions.
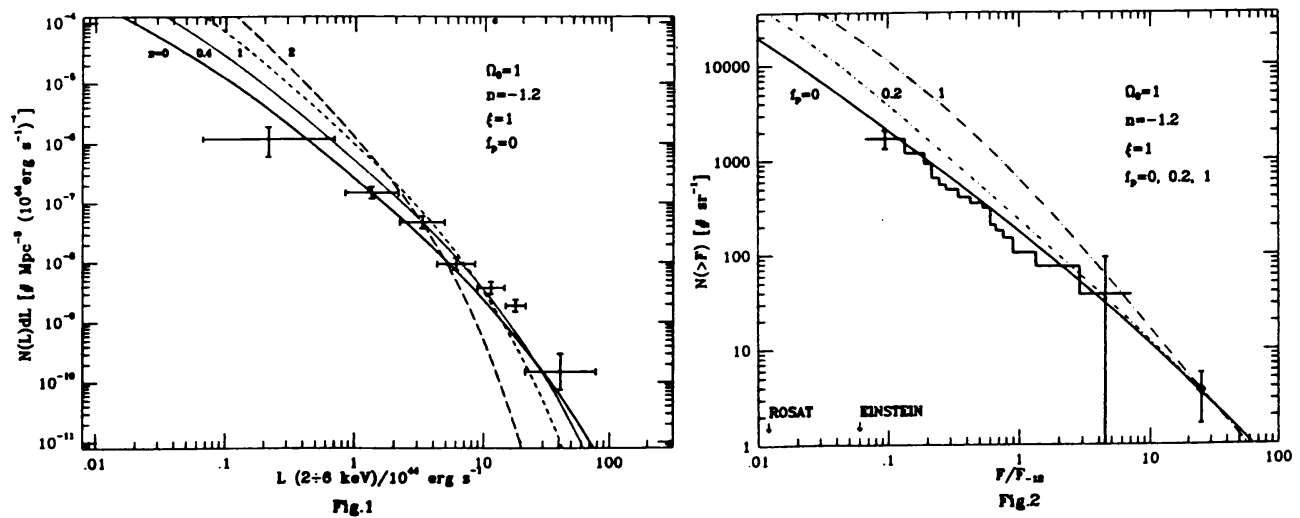

Fig. 1. The X-ray l.f. for $f_{p}=0$, with $g(t) \propto t$ (data: Johnson et al. 1983, Ap. J. 266, 425).

Fig. 2. Counts for the same model are compared with those for $f_{p}=0.2$ and $f_{p}=1$.

541

J. Audouze et al. (eds.), Large Scale Structures of the Universe, 541.

(c) 1988 by the IAU. 